\title{
Quantification of fractional flow reserve based on angiographic image data
}

\author{
Jerry T. Wong $\cdot$ Huy Le $\cdot$ William M. Suh • \\ David A. Chalyan · Toufan Mehraien • Morton J. Kern • \\ Ghassan S. Kassab · Sabee Molloi
}

Received: 19 March 2010/ Accepted: 7 December 2010/Published online: 7 January 2011

(C) The Author(s) 2010. This article is published with open access at Springerlink.com

\begin{abstract}
Coronary angiography provides excellent visualization of coronary arteries, but has limitations in assessing the clinical significance of a coronary stenosis. Fractional flow reserve (FFR) has been shown to be reliable in discerning stenoses responsible for inducible ischemia. The purpose of this study is to validate a technique for FFR quantification using angiographic image data. The study was carried out on 10 anesthetized, closed-chest swine using angioplasty balloon catheters to produce partial occlusion. Angiography based FFR was calculated from an angiographically measured ratio of coronary blood flow to arterial lumen volume. Pressure-based FFR was measured from a ratio of distal coronary pressure to aortic pressure. Pressure-wire measurements of FFR $\left(\mathrm{FFR}_{P}\right)$ correlated linearly with angiographic volume-derived measurements of FFR $\left(\mathrm{FFR}_{V}\right)$ according to the equation: $\mathrm{FFR}_{P}=0.41 \mathrm{FFR}_{V}+0.52(P$-value $<0.001)$.
\end{abstract}

J. T. Wong · H. Le - D. A. Chalyan - T. Mehraien ·

S. Molloi $(\bowtie)$

Department of Radiological Sciences, Medical Sciences I, B-140, University of California, Irvine, CA 92697, USA

e-mail: symolloi@uci.edu

W. M. Suh · M. J. Kern

Division of Cardiology, Department of Medicine,

University of California, Irvine, CA 92868, USA

G. S. Kassab

Department of Biomedical Engineering, Indiana-Purdue University, Indianapolis, IN 46202, USA
The correlation coefficient and standard error of estimate were 0.85 and 0.07 , respectively. This is the first study to provide an angiographic method to quantify FFR in swine. Angiographic FFR can potentially provide an assessment of the physiological severity of a coronary stenosis during routine diagnostic cardiac catheterization without a need to cross a stenosis with a pressure-wire.

Keywords Angiography $\cdot$ Blood flow $\cdot$ Blood volume $\cdot$ Regional blood flow $\cdot$ Stenosis

\section{Introduction}

Coronary angiography provides an assessment of stenosis severity by visualizing the opacified arterial lumen. However, assessment of stenosis severity in percent diameter correlates poorly with its physiological significance $[1,2]$. Pressure-wire measurement of fractional flow reserve (FFR) has been shown to be reliable in discerning stenoses responsible for inducible ischemia [3, 4]. Pressure-derived measurement of FFR has aided in identifying clinically relevant stenoses and evaluating the effectiveness of revascularizations [5-8].

Quantification of FFR using angiographic images would be a valuable tool in the cardiac catheterization laboratory, because coronary angiography would then 
provide both anatomical and physiological assessment of a stenosis. This would eliminate the need to advance a pressure wire across a stenotic lesion, and reduce the cost and time of the procedure. Furthermore, it will provide the opportunity to measure FFR for any lesion in the coronary arteries using only diagnostic angiographic image data. FFR is defined as the ratio of the maximum blood flow in the diseased artery to the hypothetical normal maximum blood flow in the same artery. Hyperemic coronary blood flow could be measured with angiography. Previous densitometric techniques for measuring coronary blood flow have been hampered by physical and physiological limitations. The major limitations include the non-linearity caused by physical degradation factors (such as X-ray scatter and veiling glare) and the inability to measure blood iodine concentration. However, more recent studies have addressed these limitations [9] by correcting for scatter and veiling glare and by power injection of known iodine concentration in the coronary arterial tree $[10,11]$. The technique for measuring absolute volumetric coronary blood flow has previously been validated using flow probes [10-13].

The challenge in determining FFR lies in quantifying the hypothetical normal maximum blood flow in an already diseased artery. Recent studies have shown that the maximum flow through any point in the epicardial coronary arterial tree is related to the sum of its distal coronary arterial lumen volume [1418]. Based on these studies, coronary arterial volume can be used to estimate the normal maximum blood flow. A previous study has validated an angiographic method for measuring lumen volume [19]. Therefore, FFR could be measured using angiographic images, acquired at maximum hyperemia, by quantifying both the coronary blood flow and arterial lumen volume.

The aim of this study was to determine the correlation between FFR measured with angiographic image data and FFR based on pressure-wire. A swine model was used to test the hypothesis that angiographically measured FFR is strongly correlated with pressure-derived FFR. This is the first effort to quantify FFR with angiographic image data in an in vivo model. The determination of FFR with angiography can provide valuable physiological, as well as anatomical, information about a stenosis during routine diagnostic cardiac catheterization.

\section{Methods}

In vivo swine model

A closed-chest swine model was used to measure FFR for various levels of stenosis severities in the left anterior descending (LAD) artery. For each stenosis, a set of pressure recordings and coronary angiograms were acquired. The study protocol was approved by the University of California, Irvine Institutional Animal Care and Use Committee.

Fasted swine (Yorkshire, 25-35 kg, $N=10$ ) were sedated and pre-medicated with xylazine $(2.0 \mathrm{mg} /$ $\mathrm{kg})$, ketamine $(10 \mathrm{mg} / \mathrm{kg})$, and atropine $(0.05 \mathrm{mg} / \mathrm{kg})$. Anesthesia was maintained with approximately 1-2\% isoflurane (Highland Medical Equipment Vaporizer; Temecula, CA). Heart rate and percent oxygen saturation were continuously measured (Nellcor N200 Pulse Oximeter; Hayward, CA). Arterial partial pressure of $\mathrm{CO}_{2}$ was maintained within normal limits (40-45 mmHg). Expired partial pressure of $\mathrm{CO}_{2}$ was continuously monitored with a $\mathrm{CO}_{2}$ monitor (Ohmeda 5200, Ohmeda, Liberty Corner, NJ), while arterial partial pressure of $\mathrm{CO}_{2}$ was periodically determined via arterial blood gas measurements. Carotid artery and jugular vein cut-downs were employed for sheath placement. Prior to catheterization, heparin was given (10,000 units bolus followed by additional 4,000-5,000 units/h). The left main ostium was cannulated with a $6 \mathrm{~F}$ or $7 \mathrm{~F}$ hockey-stick catheter through the left carotid artery under fluoroscopic guidance. Electrocardiogram, arterial blood pressure, and distal coronary blood pressure were continuously measured with the ComboMap (Volcano Corp; Rancho Cordova, CA). All signals measured by the ComboMap along with the X-ray pulse signal were continuously recorded and analyzed post-study with AcqKnowledge (Biopac Systems, Inc.; Santa Barbara, CA). An example of the recorded signals during resting and hyperemic flows is shown in Fig. 1.

Each swine was positioned on its right side under a flat panel detector. The projection angle was optimized for the separation of the LAD and left circumflex artery perfusion beds as performed during diagnostic cardiac catheterization. Unavoidable overlap of small branches can contribute to the error in flow and volume calculation, but these errors are small relative to the overall flow and volume. Intracoronary injection of papaverine $(5-10 \mathrm{mg})$ 


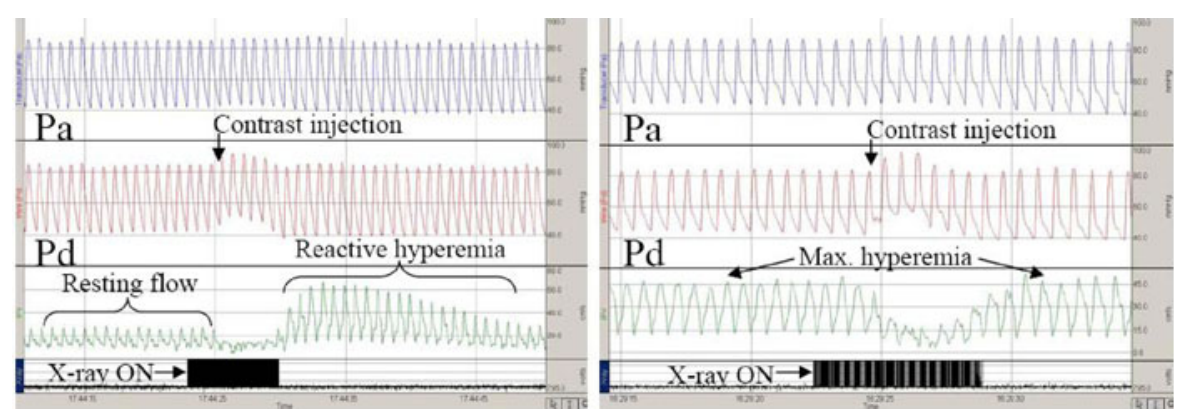

Fig. 1 Continuous recording of pressures, flow velocity, and X-ray signal during (left) resting and (right) hyperemic flow

was used for maximum hyperemic induction. Coronary angiograms were acquired within $90 \mathrm{~s}$ after intracoronary administration of papaverine. Prior to coronary angiography, pancuronium $(0.1 \mathrm{mg} / \mathrm{kg})$ was administered intravenously, and the ventilator was turned off at the end of full expiration to minimize respiratory motion. Contrast material (Omnipaque350; Princeton, NJ) was power injected (LeibelFlarsheim Angiomat 6000; Cincinnati, OH) at $4 \mathrm{ml} / \mathrm{s}$. At least a full heart cycle of images was acquired prior to contrast material injection for the selection of a cardiac phase-matched mask image for digital background subtraction. An image of a calibration phantom positioned over the heart was also acquired to determine the correlation between image gray level and iodine mass. The exclusion criterion included respiratory motion. Experimental runs with technical errors such as poor catheter engagement, complete occlusion of any branch, and acquiring angiograms later than $90 \mathrm{~s}$ after the administration of papaverine were omitted from the study.

A range of blood flow in normal swine LAD was produced with balloon catheters of different sizes and inflation pressures. Balloon sizes ranged from 2.5 to $3.75 \mathrm{~mm}$ in diameter and $8-15 \mathrm{~mm}$ in length based on a qualitative visual estimation of each LAD diameter. The coronary balloons were advanced to the proximal LAD over a guide wire under fluoroscopic guidance. The coronary balloons were then inflated to different pressures to produce a range of occlusions and hence different degrees of coronary blood flow reduction. A similar approach has been used previously [20]. The effect of stenosis severity on flow and pressure were studied. However, the degree of stenosis was not measured because placement of balloons made it difficult to accurately measure percent stenosis. The correlation between FFR and angiographic stenosis has previously been shown to be poor [21]. Each implemented stenosis was considered to be independent from each other since the purpose of the study was to determine if angiographic FFR provides a good surrogate measure of pressure-wire FFR.

Imaging system

All images were acquired using a conventional X-ray tube with a constant potential X-ray generator (Optimus M200, Philips Medical Systems, Shelton, CT). A cesium-iodide-based flat panel detector (PaxScan 4030A, Varian Medical Inc., Palo Alto, CA) was used for image acquisition. The flat panel detector has a $40 \times 30 \mathrm{~cm}^{2}$ field of view and pixel size of $0.194 \times 0.194 \mathrm{~mm}^{2}$. The zoom-center mode was used to acquire images with $1,024 \times 768$ pixels. Gain and flat field corrections were performed prior to image acquisition. The flat panel detector has no pincushion distortion and has a dynamic range greater than 8,000. Images were acquired at 30 frames/s. The detector signal in each pixel was digitized with 14-bit precision. Images were logarithmically transformed before subtraction. A Pentium IV computer and publicly available software Image J (NIH, Bethesda, MD) were used for image analysis.

\section{Angiographic based FFR}

Our goal was to measure coronary FFR using only angiographic image data. Coronary FFR is defined as follows:

$\mathrm{FFR}_{Q}=\frac{Q_{\mathrm{S}}}{Q_{\mathrm{N}}}$

where $Q_{\mathrm{S}}$ is the hyperemic flow through an artery with a stenosis, and $Q_{\mathrm{N}}$ is the hypothetical normal 
hyperemic flow through the same artery without disease. A first pass analysis technique to measure coronary blood flow using angiographic image data has previously been validated using flow probe $[10,11,22]$. Therefore, coronary blood flow in the stenotic artery $\left(Q_{\mathrm{S}}\right)$ can be directly measured using this technique. The challenge with calculating FFR is determining the hypothetical normal hyperemic flow in the same artery $\left(Q_{\mathrm{N}}\right)$. The dependent myocardial mass is used in positron emission tomography (PET) to estimate the expected normal blood flow. Myocardial mass cannot be estimated using angiography. Previous studies, however, have shown that flow through any point in the epicardial coronary arterial tree is also related to the sum of the distal coronary arterial lumen volume [14-18]. The relationship between flow $(Q)$ and the distal arterial lumen volume $(V)$ was found to be:

$Q_{\mathrm{N}}=k\left(\frac{V}{V_{\mathrm{ref}}}\right)^{3 / 4}$

where $k$ is the scaling coefficient relating crown volume to normal maximum hyperemic flow. The scaling coefficient $k$ has units of $\mathrm{ml} / \mathrm{min}$ since $Q_{\mathrm{N}}$ has units of $\mathrm{ml} / \mathrm{min}$ and lumen volume was made dimensionless by normalization to a reference volume of $V_{\text {ref }}=1 \mathrm{ml}$. By combining Eqs. 1 and 2, FFR can then be calculated using:

$\mathrm{FFR}_{V}=\frac{Q_{\mathrm{S}}}{k\left(\frac{V}{V_{\text {ref }}}\right)^{3 / 4}}$

The above equation shows that FFR can be measured using $Q_{\mathrm{S}}, V$ and $k$. A technique to measure lumen volume using angiographic image data has previously been validated [19]. Therefore, all of the parameters necessary to calculate coronary FFR can be measured using angiographic image data.

Coronary arterial volume $(V)$ was determined using angiographic images acquired during enddiastole after contrast material has propagated throughout the epicardial arteries, but before the opacification of the coronary sinus and termination of power injection. A calibration of the imaging chain for quantifying iodine mass from X-ray densitometric signal was performed by acquiring an image of the calibration phantom over the pig hearts [10, 23, 24]. The calibration phantom was placed on the chest so that its projection overlapped the heart. Correction was made for differential magnification of the phantom and the heart. Power injection of contrast material was assumed to momentarily replace blood with contrast material. The known iodine concentration in the contrast material and a linear regression analysis between measured gray levels and known iodine masses in the calibration phantom were used to convert gray level to volume. A region-of-interest (ROI) was drawn around visible arteries for volume quantification (see Fig. 2). Coronary arterial volume was then calculated using densitometry. Gray values were converted to volume measurements with a conversion factor determined from the acquired image of the calibration phantom [19].

Coronary blood flow $\left(Q_{\mathrm{S}}\right)$ was determined from the change in volume within one cardiac cycle. A global ROI encompassing the visible arteries, as well as the microcirculatory blush, was drawn for flow measurement (see Fig. 3). Coronary flow was then quantified using a first pass distribution analysis. The first pass distribution analysis assumes that contrast material of a known concentration enters a collection reservoir via a single arterial input and that all measurements are made prior to the contrast material leaving the selected region designated by the ROI. The change in the measured densitometric signal was converted to volume measurement using system

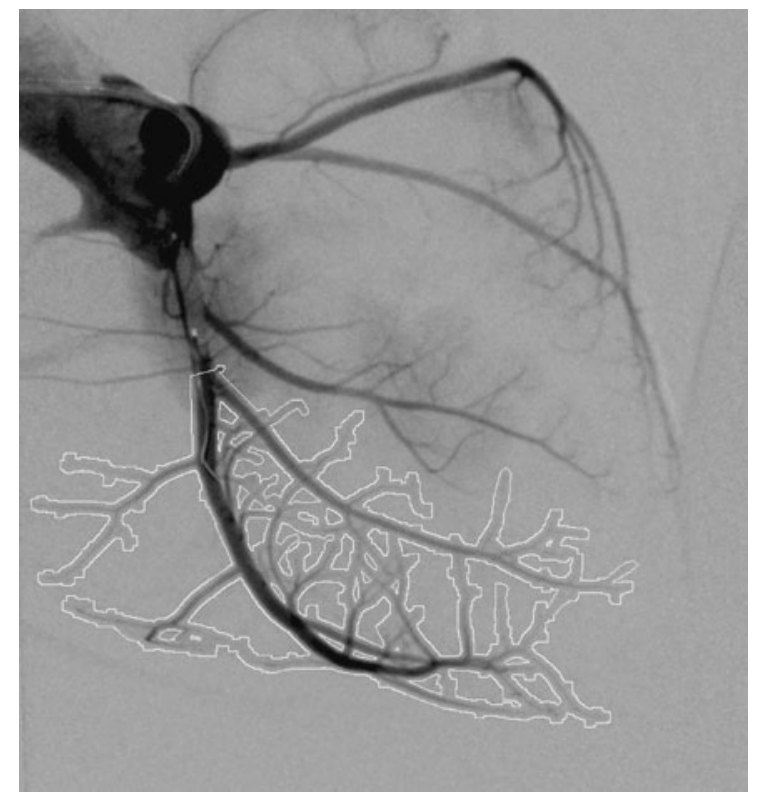

Fig. 2 An example of a region-of-interest used for angiographic volume determination in epicardial arteries 


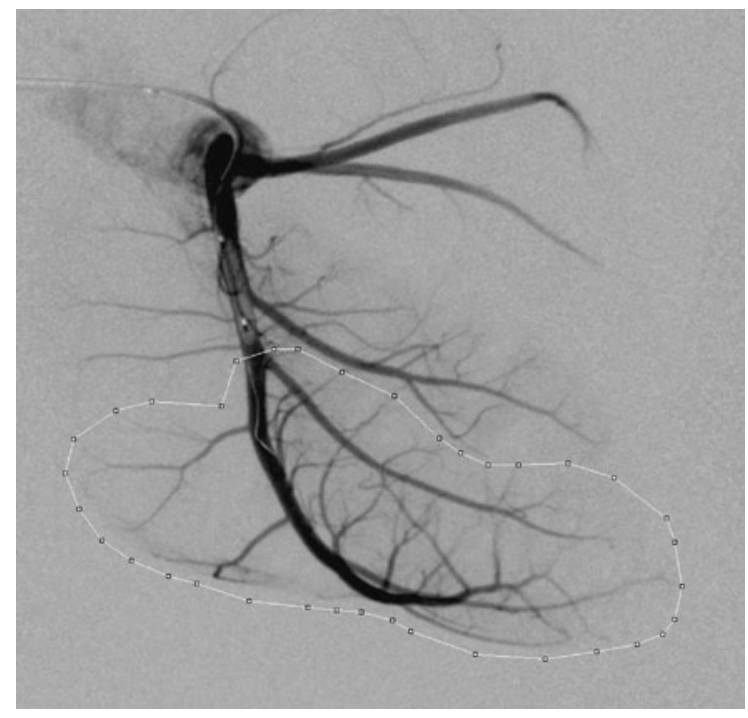

Fig. 3 An example of a global region-of-interest (ROI) used for angiographically measured coronary volume flow

iodine calibration. The time period of the cardiac cycle was calculated from the image acquisition rate of $30 \mathrm{frames} / \mathrm{s}$. The ratio of the measured volume change to the time period of the cardiac cycle yields volumetric coronary blood flow [10, 11, 22].

The influence of hemodynamic conditions (such as heart rate, mean arterial pressure, contractility, and left ventricular preload) on hyperemic blood flow that is independent of changes in the resistance state of the coronary circulation is known. Previous studies observed that hyperemic blood flow did not change significantly with heart rate [25-27], left ventricular preload [25], or ventricular contractility [26, 28]. However, hyperemic blood flow was altered proportionately with arterial pressure [25-27]. In order to address the influence of arterial pressure on hyperemic flow, the scaling coefficient (see Eq. 2) was calculated as a function of pressure according to the following:

$k=m\left(\frac{P_{\mathrm{a}}}{P_{\text {ref }}}\right)+b$

where $m$ and $b$ are the slope and $y$-intercept of the regression line, respectively. The mean aortic pressure $P_{\mathrm{a}}$ was made unitless by normalizing to a reference pressure $P_{\text {ref }}=1 \mathrm{mmHg}$. Thus, both the slope and $y$-intercept parameters have units of $\mathrm{ml} /$ min. In order to evaluate the linear correlation in
Eq. 4, coronary flow, arterial volume, and $P_{\mathrm{a}}$ during maximum hyperemia in the absence of a stenosis were measured in each pig. The values of $k$ were calculated from the measured normal coronary flow and volume, and plotted against $P_{\mathrm{a}}$. A regression analysis was then performed to determine the values of $m$ and $b$. The values for the parameters have been determined to be $m=0.99$ and $b=98.07$. Because $k$ in Eq. 4 accounts for the effects of aortic pressure on the relationship between normal maximum hyperemic coronary flow and arterial volume, coronary flow and volume measurements can be used directly to calculate $\mathrm{FFR}_{V}$ in Eq. 3 .

\section{Pressure-based FFR}

Current clinical measurement of FFR is pressurederived $\left(\mathrm{FFR}_{P}\right)$ according to the following expression using aortic pressure $\left(P_{\mathrm{a}}\right)$ and the coronary pressure distal to the stenosis $\left(P_{\mathrm{d}}\right)$ :

$\mathrm{FFR}_{P}=\frac{P_{\mathrm{d}}}{P_{\mathrm{a}}}$

This clinically used term can be further described as a measure of myocardial FFR, which takes into account collateral contribution. Intracoronary measurements of pressure were performed using a ComboWire (ComboWire Model 9515 and 9500, Volcano Corp.; Rancho Cordova, CA). The ComboWire was positioned alongside or within the balloon catheter. When inside the balloon catheter, the maneuverability of the ComboWire was diminished and a substantial drift in the pressure signal was noted when the balloon catheter shaft overrode the pressure sensor. Thus, the ComboWire was advanced so that the pressure sensor was sufficiently distal to the balloon catheter tip and care was taken not to pull the pressure sensor portion of the ComboWire to within the monorail of the balloon. Once the balloon and ComboWire were in place, equalization of pressures was performed with the balloon deflated at resting blood flow. Maximum hyperemia was induced with papaverine. $P_{\mathrm{a}}$ and $P_{\mathrm{d}}$ were measured continuously with a pressure transducer and ComboWire, respectively. $\mathrm{FFR}_{P}$ was calculated from mean pressure values over 5 cardiac cycles just prior to coronary angiography. With contrast material injection lasting for approximately $3 \mathrm{~s}$, the time between $\mathrm{FFR}_{P}$ and $\mathrm{FFR}_{V}$ measurements was at most $5 \mathrm{~s}$. 
Statistical analysis

Linear regression analysis was performed between $\mathrm{FFR}_{P}$ and $\mathrm{FFR}_{V}$ to determine the coefficients in the regression equation: $\mathrm{FFR}_{P}=m_{\mathrm{r}} \mathrm{FFR}_{V}+b_{\mathrm{r}}$, where $m_{\mathrm{r}}$ and $b_{\mathrm{r}}$ are the slope and $y$-intercept of the linear regression line. The significance of the correlation between $\mathrm{FFR}_{P}$ and $\mathrm{FFR}_{V}$ was determined, where a value of $P<0.05$ was considered to be statistically significant. The correlation coefficient $(r)$ and standard error of estimate (SEE) were determined from the linear regression analysis. SEE defines the standard deviation of the measured values from the regression line.

\section{Results}

The hemodynamic data for the study are summarized in Table 1. The mean $P_{\mathrm{a}}$ was $56.0 \pm 10.9 \mathrm{mmHg}$, and the mean heart rate was $93.1 \pm 10.5$ beats $/ \mathrm{min}$. Injection of papaverine decreased the mean arterial pressure by $26.9 \pm 9.7 \%$. The mean $\mathrm{FFR}_{P}$ and $\mathrm{FFR}_{V}$ were $0.79 \pm 0.12$ and $0.66 \pm 0.23$, respectively. A total of 82 imaging measurements were made from which 11 measurements were excluded from analysis due to respiratory motion.

A comparison of $\mathrm{FFR}_{V}$ and $\mathrm{FFR}_{P}$ measurements is given in Fig. 4. A strong correlation was observed $(r=0.85)$ with $\mathrm{SEE}=0.07$. The equation of the regression line was determined as $\mathrm{FFR}_{P}=0.41$ $\mathrm{FFR}_{V}+0.52(P$-value $<0.001)$. FFR, defined as a ratio of diseased to normal flow (Eq. 1), was also quantified since the normal flow through the LAD was known from angiographic flow measurements.

Table 1 Hemodynamic data of ten swine experiments

\begin{tabular}{llc}
\hline & Mean & $\sigma$ \\
\hline Body weight $(\mathrm{kg})$ & 31 & 4 \\
$P_{\mathrm{a}}$ (mmHg) & 56.0 & 10.9 \\
$\Delta P$ with papaverine (\%) & 26.9 & 9.7 \\
Heart rate (beats/min) & 93.1 & 10.5 \\
Papaverine-angiography time (s) & 51 & 19 \\
FFR $_{P}$ & 0.79 & 0.12 \\
FFR $_{V}$ & 0.66 & 0.23 \\
\hline
\end{tabular}

The mean value and standard deviation $(\sigma)$ of each parameter are given

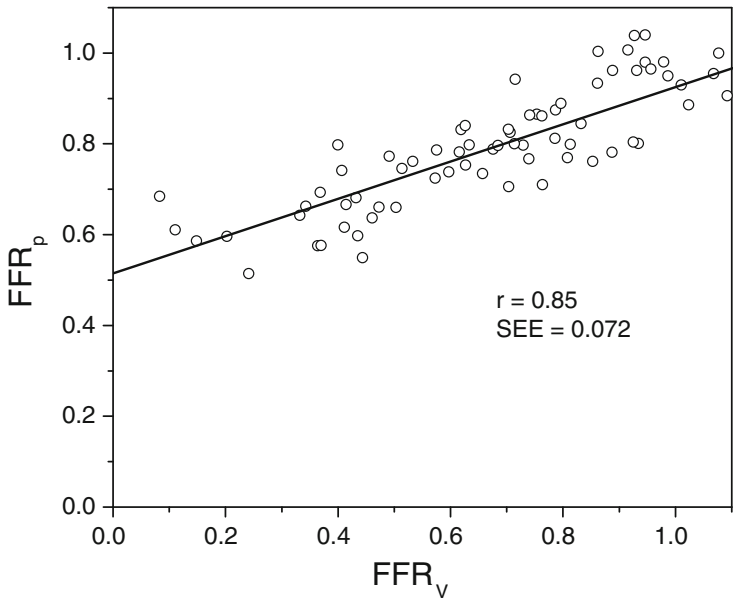

Fig. 4 A linear regression analysis of $\mathrm{FFR}_{P}$ and $\mathrm{FFR}_{V}$ measurements. The solid line represents the regression line $\left(\mathrm{FFR}_{P}=0.41 \mathrm{FFR}_{V}+0.52 ; r=0.85 ; \mathrm{SEE}=0.072\right)$. Standard errors in the slope and $y$-intercept values are 0.03 and 0.02 , respectively

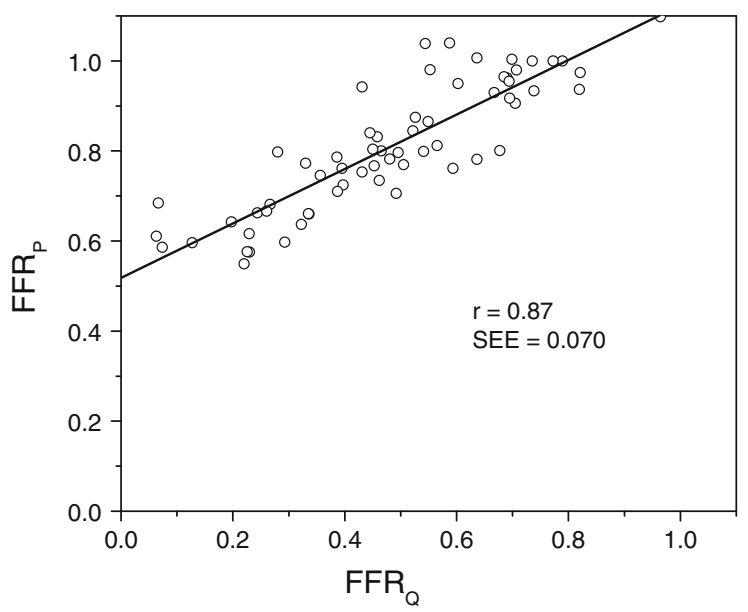

Fig. 5 A linear regression analysis of $\mathrm{FFR}_{P}$ and $\mathrm{FFR}_{Q}$ measurements. The solid line represents the regression line $\left(\mathrm{FFR}_{P}=0.61 \mathrm{FFR}_{Q}+0.52 ; r=0.87 ; \mathrm{SEE}=0.070\right)$. Standard errors in the slope and $y$-intercept values are 0.03 and 0.02 , respectively

This flow-derived FFR $\left(\mathrm{FFR}_{Q}\right)$ was calculated in the nine pigs with normal flows that were measurable with angiographic data (Pig 7 was excluded because of respiratory motion). Figure 5 compares the flowderived FFR $\left(\mathrm{FFR}_{Q}\right)$ to $\mathrm{FFR}_{P}$. The equation of the regression line relating $\mathrm{FFR}_{Q}$ to $\mathrm{FFR}_{P}$ was determined as $\mathrm{FFR}_{P}=0.61 \mathrm{FFR}_{Q}+0.52$ with a correlation coefficient of 0.87 and SEE of 0.07 . 


\section{Discussion}

The results of FFR measurements in swine showed a strong linear correlation between the proposed angiographic technique and the clinical pressure-wire method. Figure 4 shows that volume-derived FFR values measured angiographically correlated well with the pressure-derived values measured with a pressure sensor-wire $(r=0.85$ and $\mathrm{SEE}=0.07)$. These experimental results were similar to previous studies comparing pressure-derived FFR to relative flow reserve (RFR) determined with PET [3, 29]. RFR was defined as the maximal achievable perfusion in the stenotic region divided by the maximal achievable perfusion in the neighboring region supplied by a normal coronary artery. Under similar microvascular resistances and hemodynamic conditions, RFR should be equivalent to FFR. De Bruyne et al. [3] showed that pressure-derived myocardial FFR correlated closely with RFR measured with PET, with $r=0.87$ and $\mathrm{SEE}=0.13$. Similarly, Marques et al. [29], in their study of the effects of myocardial infarction on microvascular resistance, found that FFR and RFR correlated well $(r=0.81)$. Their measurements of RFR and FFR showed a mean difference of 0.01 with a standard deviation of 0.11 .

Figure 4 shows that $\mathrm{FFR}_{V}$ underestimated $\mathrm{FFR}_{P}$ at lower values. A similar underestimation was observed by Pijls et al. [30] when they compared coronary FFR as defined by a flow ratio to myocardial FFR calculated by a pressure ratio. Pijls et al. argued that the observed difference was due to the inclusion of collateral flow in myocardial FFR but not in coronary FFR, where $y$-intercept values as high as 0.36 were attributed to the abundant collateral circulation in mongrel dogs, but the collateral circulation were not verified with direct measurement. Collateral circulation in swine is known to be negligible; thus, coronary and myocardial FFR should be similar in pigs, but Fig. 4 shows that $\mathrm{FFR}_{V}$ underestimated $\mathrm{FFR}_{P}$. An argument against collateral circulation as a substantial source of difference in measured FFR values in the range of clinically applicable perfusion pressures and moderate stenosis levels includes the study by Messina et al. [31] where differences in flow with and without collateral circulation were only appreciable at low perfusion pressures $(<40 \mathrm{mmHg})$. This suggested that FFR measured by a flow ratio with and without collateral circulation is similar for perfusion pressures greater than $40 \mathrm{mmHg}$. Thus, the differences observed by Pijls et al. between coronary and myocardial FFR in dogs cannot be fully attributed to collateral circulation alone. Additionally, when myocardial FFR values based on perfusion measurements were compared to pressure measurements an underestimation of perfusion measurements relative to pressure measurements was observed [3]. Since the contribution of collateral flow would be included in perfusion measurements, the observed underestimation would not be due to collateral circulation.

Instead, the difference in FFR measurements may be explained by the inherent theoretical differences between the ratio $P_{\mathrm{d}} / P_{\mathrm{a}}$ and $Q_{\mathrm{S}} / Q_{\mathrm{N}}$ and the nonlinear relationship between flow and pressure changes. A comparison of $\mathrm{FFR}_{P}$ and $\mathrm{FFR}_{V}$ should not be expected to follow the line of identity because the ratio of $P_{\mathrm{d}} / P_{\mathrm{a}}$ is not equivalent to $Q_{\mathrm{S}} / Q_{\mathrm{N}}$ if back pressure in terms of wedge pressure is ignored. Wedge pressure can reach as high as $25 \mathrm{mmHg}$ in the absence of any collateral flow. Together with the considerably low mean aortic pressure $\left(P_{\mathrm{a}}=56 \mathrm{mmHg}\right)$ in our animals, the lack of correction for $P_{\mathrm{w}}$ could introduce significantly large $y$ intercept values.

The observed highly correlated, but non-identical, relationship between $\mathrm{FFR}_{P}$ and $\mathrm{FFR}_{V}$ in Fig. 4 is in accordance with flow-pressure relationships. For example, previous studies have shown that the coronary pressure-flow curve is convex rather than linear, where flow drops off more rapidly than pressure at low pressure or flow states [32]. The observed nonlinearity stems from the compliance or elasticity of coronary vessels as they respond to the decreasing distending pressures distal to the stenosis [33]. Figure 6 plots the data from Pantely et al. [32, 34, 35] in the form of pressure- and flow-derived FFR values. The FFR values plotted in Fig. 6 were determined using their raw distal pressure and flow measurements normalized by maximum pressure and flow measurements to reflect $P_{\mathrm{a}}$ and $Q_{\mathrm{N}}$ values, respectively. Figure 6 clearly shows the nonlinearly between $P_{\mathrm{d}} / P_{\mathrm{a}}$ and $Q_{\mathrm{S}} / Q_{\mathrm{N}}$. The experimentally measured $\mathrm{FFR}_{P}$ (which is $P_{\mathrm{d}} / P_{\mathrm{a}}$ ) and $\mathrm{FFR}_{Q}$ (which is equivalent to $Q_{\mathrm{S}} / Q_{\mathrm{N}}$ ) were also included in Fig. 6 in order to demonstrate that the experimentally observed relationship is consistent with previous flow and pressure results. Figure 6 also shows that the relationship between $P_{\mathrm{d}} / P_{\mathrm{a}}$ and $Q_{\mathrm{S}} / Q_{\mathrm{N}}$ is approximately linear for values greater 0.2 , which is important since coronary stenoses with FFR values less 


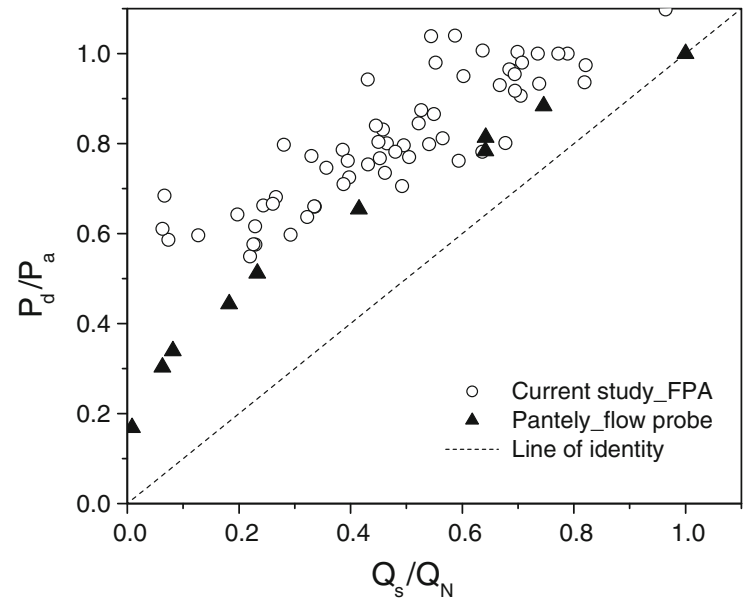

Fig. 6 Pooled data showing the relation between simplified myocardial fractional flow reserve $\left(P_{\mathrm{d}} / P_{\mathrm{a}}\right)$ and direct flow reserve $\left(Q_{\mathrm{S}} / Q_{\mathrm{N}}\right)$ in swine. Open circles represent data from the current study where $\mathrm{FFR}_{P}$ is $\left(P_{\mathrm{d}} / P_{\mathrm{a}}\right)$ and $\mathrm{FFR}_{Q}$ is equivalent to $\left(Q_{\mathrm{S}} / Q_{\mathrm{N}}\right)$. Closed triangles are extracted from published data by Pantely et al. [32, 34, 35] where blood flow was measured with Doppler flow probe

than 0.2 do not require FFR measurement for intervention. An extrapolation of data in this linear portion would lead to the observed $0.49 y$-intercept. Previous studies have found similar overestimations of the pressure intercept when considering the same range of pressures and flows [32, 34, 35].

In comparing our measured $\mathrm{FFR}_{Q}$ to $\mathrm{FFR}_{P}$ we see a slightly higher correlation $(r=0.87)$ than we do for $\mathrm{FFR}_{V}$. This is expected, since normal hyperemic flow is measured directly rather than estimated from arterial volume. However, the rather small difference in errors between Figs. 4 and 5 suggests that volume-based estimation of normal hyperemic flow adds only limited variability and can be used in the clinical setting, where normal flow through a diseased artery is not available.

Given the limitations of uncorrected pressurebased FFR calculation, future clinical studies might benefit from direct flow measurement of the imagebased technique. Ideally, all future comparisons should be referenced to another blood flow modality, potentially PET or flow probe (if done in animals). However, pressure-based comparisons are sufficient if an accurate measure of wedge pressure is available.

\section{Study limitations}

Measurement errors associated with angiographic measurements of coronary volume and flow are higher than pressure measurements by sensor-wire. Where pressure measurements are known to be highly reproducible, the variability in volume measurement was approximately $9 \%$ and variability in coronary flow was approximately $15 \%$ when compared to Doppler sensor-wire measurements of average peak velocity. The reproducibility of $\mathrm{FFR}_{V}$ was not assessed, but the reproducibility of volume and flow measurements have been previously studied [10, 19].

Additionally, $\mathrm{FFR}_{V}$ values can be greater than 1 because the expected flow in absence of a stenosis is estimated based on the measured volume using the equation for the scaling coefficient (see Eq. 3). Values greater than 1 are due to measurement error as well as variability in the scaling coefficient $k$. The inherent heterogeneity in $k$ can affect the clinical effectiveness of $\mathrm{FFR}_{V}$ for evaluating stenosis severity. In the current study, a variability of approximately $10 \%$ was observed in $k$, which was comparable to previous studies on the heterogeneity in hyperemic perfusion using PET [36, 37]. Thus, the clinical application of this methodology will require a reassessment of the scaling coefficient expressed in Eq. 4 as well as a study on the threshold for hemodynamically significant stenoses similar to that validated for $\mathrm{FFR}_{P}$. A simple estimation using the regression equation shown in Fig. 4 suggests a threshold of approximately $\mathrm{FFR}_{V}=0.70$ for a predetermined $\mathrm{FFR}_{P}$ threshold of 0.80 , but a validation study will be required in a clinical setting.

In the current study, only coronary angiograms without respiratory motion were analyzed for $\mathrm{FFR}_{V}$. However, images without respiratory motion cannot always be expected in the clinical setting. Respiratory motion can introduce misregistration artifacts in phase-matched subtracted images and increase measurement error in coronary flow and volume. However, motion misregistration artifacts can be minimized through breath-hold due to the short time interval required for image acquisition (3-5 s), as compared to a previously reported technique that required 15-20 s [38]. In addition, a technique of using linear interpolation to measure coronary flow and volume signals in unsubtracted images has been developed [24].

Another limitation of the current study is that only LADs in normal coronary networks from healthy swine were studied. The experimental model only 
simulated a focal epicardial stenosis. Other disease conditions, such as ventricular hypertrophy, diffuse coronary artery disease, and prior myocardial infarction can potentially introduce disparities between $\mathrm{FFR}_{V}$ and the level of epicardial stenosis severity. The influence of other disease conditions on $\mathrm{FFR}_{V}$ requires additional study. Moreover, since only LADs were studied, different results using different coronary territories is potentially possible.

\section{Conclusions}

This study validated a linear relationship between an angiographic FFR and the standard pressure-derived FFR. The application of angiographic FFR in humans would provide a useful method of assessing the physiological severity of a coronary stenosis during diagnostic cardiac catheterization without a need to cross a stenosis with a pressure-wire. Therefore, angiographic images can potentially be used for both anatomical and physiological assessment of coronary artery disease.

Acknowledgments The authors wish to thank David Wong and Mark Lete of Volcano Corporation for their technical support with the ComboMap system. Jerry Wong and Huy Le were supported through the National Institute of Health (NIH) Medical Scientist Training Program MD/PhD training grant at the University of California, Irvine. This research is supported in part by the NIH Grant R01HL089941 and the SCAI/Cordis Fellowship Program for Interventional Cardiology.

\section{Conflict of interest None.}

Open Access This article is distributed under the terms of the Creative Commons Attribution Noncommercial License which permits any noncommercial use, distribution, and reproduction in any medium, provided the original author(s) and source are credited.

\section{References}

1. White CW, Wright CB, Doty DB et al (1984) Does visual interpretation of the coronary arteriogram predict the physiologic importance of a coronary stenosis? N Engl J Med 310:819-824

2. Folland ED, Vogel RA, Hartigan P et al (1994) Relation between coronary artery stenosis assessed by visual, caliper, and computer methods and exercise capacity in patients with single-vessel coronary artery disease. The veterans affairs ACME investigators. Circulation 89:2005-2014
3. De Bruyne B, Baudhuin T, Melin JA et al (1994) Coronary flow reserve calculated from pressure measurements in humans. Validation with positron emission tomography. Circulation 89:1013-1022

4. Pijls NH, De Bruyne B, Peels K et al (1996) Measurement of fractional flow reserve to assess the functional severity of coronary-artery stenoses. N Engl J Med 334:1703-1708

5. Bech GJ, De Bruyne B, Bonnier HJ et al (1998) Long-term follow-up after deferral of percutaneous transluminal coronary angioplasty of intermediate stenosis on the basis of coronary pressure measurement. J Am Coll Cardiol 31:841-847

6. Bech GJ, De Bruyne B, Pijls NH et al (2001) Fractional flow reserve to determine the appropriateness of angioplasty in moderate coronary stenosis: a randomized trial. Circulation 103:2928-2934

7. Berger A, Botman KJ, MacCarthy PA et al (2005) Longterm clinical outcome after fractional flow reserve-guided percutaneous coronary intervention in patients with multivessel disease. J Am Coll Cardiol 46:438-442

8. Wongpraparut N, Yalamanchili V, Pasnoori V et al (2005) Thirty-month outcome after fractional flow reserve-guided versus conventional multivessel percutaneous coronary intervention. Am J Cardiol 96:877-884

9. Ersahin A, Molloi S, Yao-Jin Q (1995) A digital filtration technique for scatter-glare correction based on thickness estimation. IEEE Trans Med Imaging 14:587-595

10. Molloi S, Bednarz G, Tang J, Zhou Y, Mathur T (1998) Absolute volumetric coronary blood flow measurement with digital subtraction angiography. Int J Card Imaging 14:137-145

11. Molloi S, Zhou Y, Kassab GS (2004) Regional volumetric coronary blood flow measurement by digital angiography: in vivo validation. Acad Radiol 11:757-766

12. Marinus H, Buis B, van Benthem A (1990) Pulsatile coronary flow determination by digital angiography. Int J Card Imaging 5:173-182

13. Hangiandreou N, Folts J, Peppler W, Mistretta C (1991) Coronary blood flow measurement using an angiographic first pass distribution technique: a feasibility study. Med Phys 18:947-954

14. West GB, Brown JH, Enquist BJ (1997) A general model for the origin of allometric scaling laws in biology. Science 276:122-126

15. Kassab GS (2005) Scaling laws of vascular trees: of form and function. Am J Physiol Heart Circ Physiol 290:H894H903

16. Molloi S, Wong JT (2007) Regional blood flow analysis and its relationship with arterial branch lengths and lumen volume in the coronary arterial tree. Phys Med Biol 52:1495-1503

17. Choy JS, Kassab GS (2008) Scaling of myocardial mass to flow and morphometry of coronary arteries. J Appl Physiol 104:1281-1286

18. Wong JT, Molloi S (2008) Determination of fractional flow reserve (FFR) based on scaling laws: a simulation study. Phys Med Biol 53:3995-4011

19. Molloi S, Kassab GS, Zhou Y (2001) Quantification of coronary artery lumen volume by digital angiography: in vivo validation. Circulation 104:2351-2357 
20. Roy AS, Back MR, Khoury SF et al (2008) Functional and anatomical diagnosis of coronary artery stenoses. J Surg Res 150:24-33

21. Lim MJ, Kern MJ (2006) Coronary pathophysiology in the cardiac catheterization laboratory. Curr Probl Cardiol 31:493-550

22. Molloi S, Ersahin A, Tang J, Hicks J, Leung CY (1996) Quantification of volumetric coronary blood flow with dual-energy digital subtraction angiography. Circulation 93:1919-1927

23. Molloi SY, Mistretta CA (1989) Quantification techniques for dual-energy cardiac imaging. Med Phys 16:209-217

24. Wong JT, Kamyar F, Molloi S (2007) Quantitative coronary angiography using image recovery techniques for background estimation in unsubtracted images. Med Phys 34:4003-4015

25. McGinn AL, White CW, Wilson RF (1990) Interstudy variability of coronary flow reserve. Influence of heart rate, arterial pressure, and ventricular preload. Circulation 81: 1319-1330

26. De Bruyne B, Bartunek J, Sys SU, Pijls NH, Heyndrickx GR, Wijns W (1996) Simultaneous coronary pressure and flow velocity measurements in humans. Feasibility, reproducibility, and hemodynamic dependence of coronary flow velocity reserve, hyperemic flow versus pressure slope index, and fractional flow reserve. Circulation 94:1842-1849

27. Rossen JD, Winniford MD (1993) Effect of increases in heart rate and arterial pressure on coronary flow reserve in humans. J Am Coll Cardiol 21:343-348

28. Cleary RM, Ayon D, Moore NB, DeBoe SF, Mancini GB (1992) Tachycardia, contractility and volume loading alter conventional indexes of coronary flow reserve, but not the instantaneous hyperemic flow versus pressure slope index. J Am Coll Cardiol 20:1261-1269

29. Marques KM, Knaapen P, Boellaard R, Lammertsma AA, Westerhof N, Visser FC (2007) Microvascular function in viable myocardium after chronic infarction does not influence fractional flow reserve measurements. J Nucl Med 48:1987-1992
30. Pijls NH, van Son JA, Kirkeeide RL, De Bruyne B, Gould KL (1993) Experimental basis of determining maximum coronary, myocardial, and collateral blood flow by pressure measurements for assessing functional stenosis severity before and after percutaneous transluminal coronary angioplasty. Circulation 87:1354-1367

31. Messina LM, Hanley FL, Uhlig PN, Baer RW, Grattan MT, Hoffman JI (1985) Effects of pressure gradients between branches of the left coronary artery on the pressure axis intercept and the shape of steady state circumflex pressure-flow relations in dogs. Circ Res 56:11-19

32. Spaan JA, Piek JJ, Hoffman JI, Siebes M (2006) Physiological basis of clinically used coronary hemodynamic indices. Circulation 113:446-455

33. Huo Y, Kassab GS (2009) Effect of compliance and hematocrit on wall shear stress in a model of the entire coronary arterial tree. J Appl Physiol 107:500-505

34. Klocke FJ, Weinstein IR, Klocke JF et al (1981) Zero-flow pressures and pressure-flow relationships during single long diastoles in the canine coronary bed before and during maximum vasodilation. Limited influence of capacitive effects. J Clin Invest 68:970-980

35. Pantely GA, Ladley HD, Bristow JD (1984) Low zero-flow pressure and minimal capacitance effect on diastolic coronary arterial pressure-flow relationships during maximum vasodilation in swine. Circulation 70:485-494

36. Chareonthaitawee P, Kaufmann PA, Rimoldi O, Camici PG (2001) Heterogeneity of resting and hyperemic myocardial blood flow in healthy humans. Cardiovasc Res 50:151-161

37. Kaufmann PA, Gnecchi-Ruscone T, Yap JT, Rimoldi O, Camici PG (1999) Assessment of the reproducibility of baseline and hyperemic myocardial blood flow measurements with 15O-labeled water and PET. J Nucl Med 40:1848-1856

38. Pijls NH, Uijen GJ, Hoevelaken A et al (1990) Mean transit time for the assessment of myocardial perfusion by videodensitometry. Circulation 81:1331-1340 